\title{
Reduction in the proportion of fevers associated with Plasmodium falciparum parasitaemia in Africa: a systematic review
}

\author{
Valérie D'Acremont ${ }^{1,2,3^{*}}$, Christian Lengeler ${ }^{1}$, Blaise Genton ${ }^{1,3}$
}

\begin{abstract}
Background: Malaria is almost invariably ranked as the leading cause of morbidity and mortality in Africa. There is growing evidence of a decline in malaria transmission, morbidity and mortality over the last decades, especially so in East Africa. However, there is still doubt whether this decline is reflected in a reduction of the proportion of malaria among fevers. The objective of this systematic review was to estimate the change in the Proportion of Fevers associated with Plasmodium falciparum parasitaemia (PFPf) over the past 20 years in sub-Saharan Africa.

Methods: Search strategy. In December 2009, publications from the National Library of Medicine database were searched using the combination of $16 \mathrm{MeSH}$ terms.

Selection criteria. Inclusion criteria: studies 1) conducted in sub-Saharan Africa, 2) patients presenting with a syndrome of 'presumptive malaria', 3) numerators (number of parasitologically confirmed cases) and denominators (total number of presumptive malaria cases) available, 4) good quality microscopy.

Data collection and analysis. The following variables were extracted: parasite presence/absence, total number of patients, age group, year, season, country and setting, clinical inclusion criteria. To assess the dynamic of PFPf over time, the median PFPf was compared between studies published in the years $\leq 2000$ and $>2000$.

Results: 39 studies conducted between 1986 and 2007 in 16 different African countries were included in the final analysis. When comparing data up to year 2000 (24 studies) with those afterwards (15 studies), there was a clear reduction in the median PFPf from 44\% (IQR 31-58\%; range 7-81\%) to 22\% (IQR 13-33\%; range 2-77\%). This dramatic decline is likely to reflect a true change since stratified analyses including explanatory variables were performed and median PFPfs were always lower after 2000 compared to before.

Conclusions: There was a considerable reduction of the proportion of malaria among fevers over time in Africa. This decline provides evidence for the policy change from presumptive anti-malarial treatment of all children with fever to laboratory diagnosis and treatment upon result. This should insure appropriate care of non-malaria fevers and rationale use of anti-malarials.
\end{abstract}

\section{Background}

Currently, the global target for malaria control is to provide prompt and effective treatment as well as insecticide-treated nets (ITNs) to $80 \%$ of the people at risk of malaria by the end of 2010 [1,2]. The greatly increased malaria control effort since 2000 has been supported by global initiatives such as the Global Fund to fight AIDS, Tuberculosis and Malaria, the World Bank Malaria

\footnotetext{
* Correspondence: valerie.dacremont@unibas.ch

${ }^{1}$ Swiss Tropical and Public Health Institute, P.O. Box, 4002 Basel, Switzerland Full list of author information is available at the end of the article
}

Booster Programme and the US Presidential Malaria Initiative. By the end of 2008, more than 50 African states had adopted artemisinin-combination therapy (ACT) and the number of ITNs distributed had increased more than 10 times in 14 African states [3]. There is evidence now of reduced malaria transmission, morbidity and mortality in locations where these strategies have been massively deployed [4,5]. There is also a documented decline in Africa of Plasmodium falciparum prevalence rates in children aged 2-10 years from $37 \%$ before the year 2000, to $17 \%$ after 2000 [6]. This decline is further evidenced in recent Demographic 
and Health Surveys (DHS) in malaria endemic countries of sub-Saharan Africa: 11 of the 12 national surveys conducted since 2004 showed declines in underfive mortality estimates over the previous five years (declines of $5 \%$ to $30 \%$, median $23 \%$ ) [7].

Theoretically, a reduction of malaria transmission, and hence parasitaemia, should translate into a decline of the proportion of fevers due to malaria, but the relationship between these two parameters is not straightforward. In part, this is due to the fact that the pattern of other causes of fever found in each area or patient population is not uniform and influence, therefore, the magnitude of the effect. Annual episodes of fever among African children have been estimated to be as high as 870 million [8]. For those patients who reach clinics across the continent, a presumptive diagnosis of malaria is done in $30-40 \%$ of the cases [9]. Malaria thus appears to be the number one cause of fever in sub-Saharan Africa, as well as the leading cause of mortality, at least in children [10].

However, these data might be largely overestimated nowadays due to the lack of specificity of a purely clinical diagnosis. Assigning malaria as a cause of fever in the absence of laboratory diagnosis is based on clinical experience but also on an understanding of the underlying epidemiology of the disease. Unfortunately, current practice by health care providers largely ignores the declining trend of P. falciparum parasite prevalence observed in community surveys and the proportion of fevers attributed to malaria does not seem to change. This question is of great practical relevance to correctly estimate the burden of disease due to malaria and for tracking progress in malaria control.

The objective of the present project was, therefore, to review the available evidence on the proportion of fevers due to malaria over the last 20 years in sub-Saharan Africa, to identify trends and to quantify the magnitude of expected changes. This assessment has obvious implications for optimizing recommendations concerning the management of fever cases in children under five years of age who live in highly endemic areas.

\section{Methods}

\section{Criteria for considering studies for this review} Type of studies: observational studies or diagnostic studies Inclusion criteria were i) study conducted in an area of sub-Saharan Africa where P. falciparum is the dominant species, ii) including patients (or a clear subset of patients) presenting at a health facility with a syndrome of 'presumptive malaria', either considered as such by the health worker in charge, or defined on clinical criteria by the investigators, iii) numerator (number of parasitologically-confirmed cases) and denominator (total number of presumptive malaria cases) available or possible to calculate from text, tables, or obtained after request to the authors, iv) good quality microscopy (reference centre or research laboratory), v) no obvious selection bias of patients.

Exclusion criteria were: i) studies using a parasite density threshold for malaria case definition, ii) intervention studies and studies aimed at evaluating the incidence of malaria episodes, iii) studies including $<100$ patients, or those focusing on inpatients only and severe malaria. The reasons for excluding these studies are as follows: i) defining a parasite threshold leads to a patients population that has lower proportion of malaria than when presence/absence of parasitaemia is used to define a case, ii) messages given to subjects in interventions studies ('attend as soon as possible in case of any symptom') lead to a patients population that is likely to be biased towards milder cases, and hence potentially lower prevalence of parasitaemia (undetectable parasitaemia because of low density), iii) studies with $<100$ patients would have lacked precision (more than $+/-10 \%$ in the prevalence estimate) and be at higher risk of bias and confounding because of the limited sampling.

\section{Type of participants}

Patients of any age

\section{Type of outcome measures}

Proportion of fevers associated with Plasmodium falciparum parasitaemia (PFPf).

\section{Search method for identification of studies}

All relevant published studies of human medicine, regardless of language were searched for. In the COCHRANE reviews, 'malaria' as search term was used. In MEDLINE, a combination of several MeSH terms was used in the following way: malaria AND Africa AND (diagnosis OR parasitemia OR microscopy) AND (epidemiology OR sensitivity and specificity OR prevalence OR seasons OR transmission OR cross-sectional studies OR predictive value of tests) AND (aetiology OR fever OR algorithms OR case management). Titles and abstracts to be reviewed were listed. On the basis of abstract reading, full papers were selected, reviewed and those that matched the selection criteria were retained. Then, abstracts of the related articles of this first series of papers were explored, reviewed and eventually the full paper was read if deemed appropriate. All references of the retained papers were also examined. This process was performed iteratively until no new suitable study could be found.

\section{Databases}

Cochrane Infectious Diseases Group Specialized Register (up to $15^{\text {th }}$ December 2009), MEDLINE (through $15^{\text {th }}$ December 2009).

\section{Researchers and organizations}

The following authors were contacted for clarifications on entry criteria of patients or for additional 
information on proportion of malaria among their sample: Pedro Alonso, Patrick Kachur, Christoph Hatz, Sophie Yacoub, Babacar Faye, Thomas Mschana and Nadjitolnan Othingué.

\section{Reference lists}

The reference lists of all studies identified by the above methods was checked.

\section{Methods of the review Study selection}

One author (VdA) independently applied the inclusion criteria to all identified studies. All studies selected were checked by a second author (BG) for appropriateness. For studies for which there were doubts about inclusion, the second author assessed them fully and potential differences were discussed until consensus was reached.

\section{Data extraction}

Besides the proportion of fevers with associated Plasmodium falciparum, eight variables were extracted from each paper: year, season (rainy versus dry; when season was not mentioned it was searched on the CIA website [11] using dates of beginning and end of recruitment), country, setting (urban versus rural), health facility type (hospital versus primary care), age group ( $<5$ years, 5 15 years, adults), clinical inclusion criteria used and total number of patients.

\section{Data analysis}

As it was not possible to obtain the original databases of all studies, essentially a descriptive analysis on aggregated data was performed. The proportion of fever or presumptive malaria cases associated with $P$. falciparum documented by high quality microscopy, hereinafter referred to as proportion of fevers due to malaria (PFPf), was retrieved or calculated from each study. To assess the trend of PFPf over time, the median PFPf including all studies (pooled analysis) was compared for the period up to the year 2000, and for that from 2001 onwards. This threshold was chosen since large-scale interventions started around this time thanks to a massive increase in funding for control measures [12]. That cut-off is also consistent with the analysis by Guerra et al [6] for parasitaemia. No formal multivariate analysis was possible since individual records for each study were not available. However, to investigate potential confounding factors, a stratified analysis by age group ( $<5$ and $\geq 5$ years), season (rainy and dry), setting (rural and urban), type of health facility (primary care and hospital) was performed using data from studies where such information was available. These parameters are known to have an effect on malaria fevers and the categories are standard to describe the epidemiology of malaria.

\section{Results}

\section{Description of studies}

Up to $15^{\text {th }}$ December 2009, 170 titles were extracted from the COCHRANE database, but none was relevant for this review. From MEDLINE, 524 titles were identified and extracted, all abstracts were reviewed and 41 papers were selected in the first round. Based on the reading of the full article, 20 met all inclusion criteria and were retained. After iterative cross-referencing of these 20 articles, 19 additional articles meeting all inclusion criteria were found (see Figure 1). The 39 studies included in the final analysis were conducted between 1986 and 2007 and published between 1989 and 2009. The total number of patients included was 42,979 (median: 576; range: 149 to 7713). All relevant details of the included studies are described in Table 1.

\section{Time frame}

24 studies were conducted up to the year 2000 and 15 from 2001 onwards.

\section{Location}

The included studies were conducted in 16 different African countries. 21 studies took place in East Africa, mainly Tanzania (12 studies), 16 studies in West Africa, one in Central Africa (Chad) and one in the Northern region (Sudan) (Figure 2).

\section{Age group}

Fifteen studies included children only (age $<5$ years, $\mathrm{n}=$ 8 ; age $<9$ years, $\mathrm{n}=6$; age $<12$ years, $\mathrm{n}=1$ ). One study included only adults older than 18 years and one study only patients older than 5 years. The remaining 22 studies included patients of all ages.

\section{Level of endemicity and transmission season}

Studies on clinical management of malaria have been done mainly in highly endemic areas and a period of the year with peak transmission, and this is reflected in the included studies. Patients were recruited during the rainy season only, the rainy and the dry seasons and the dry season only in 19, 16 and 4 studies, respectively.

\section{Setting and level of health care}

Twenty one studies were conducted in a rural area of Africa (54\%), while the remaining took place in urban areas. Also, 21 studies were undertaken at primary care level and the rest at hospital level.

\section{Overall proportion of parasitaemia among fever cases (PFPf)}

The proportion of parasitaemia among fever or presumptive malaria cases (PFPf) varied considerably between studies and sub-groups of patients within the same study. Taking each study as a unit, the median PFPf was 35\% [inter-quartile range - IQR: 20-54\%; range $2-81 \%)$. 


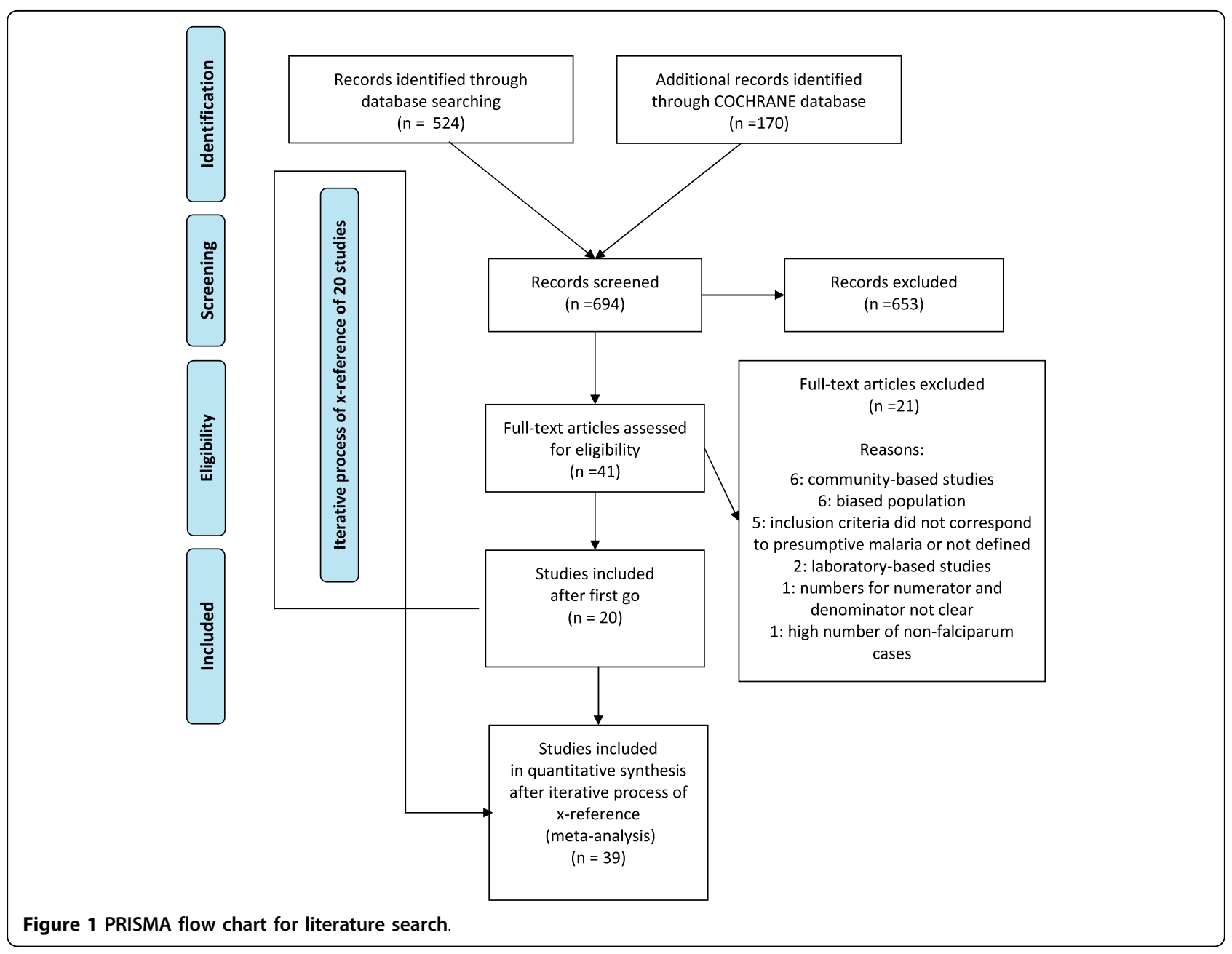

\section{Variation of PFPf according to key stratification parameters \\ Age group-specific PFPf}

Taking into account all studies where data were stratified by age or those that included only one defined age group, overall median PFPf was 36\% (IQR 21-60\%; range $4-77 \%$ ) for children under five years (25 studies) and $26 \%$ (IQR 13-33\%; range 1-53\%) for those above five years (18 studies). In the 16 studies providing stratified values of PFPf in both age groups, median PFPf in children under five years of age (32\%; IQR 18-55\%) was not significantly different from that in the group above five years (27\%; IQR 19-34\%). When using only the 10 studies providing details for the older age groups, the median PFPf was 27\% (IQR 20-50\%) for the group under 5 years, $40 \%$ (IQR 22-48\%) for the age group of $5-15$ years, and 24\% (IQR 11-27\%) in adults above 15 years.

\section{Season-specific PFPf}

The overall median PFPf was 37\% (IQR 30-60\%; range $4-77 \%$ ) in the rainy season versus 5\% (IQR 4-12\%; range
0-28\%) in the dry season. Among all factors studied, this was the most dramatic difference observed. This difference remained when restricting the analysis to the $7 \mathrm{stu}$ dies providing stratified value of PFPf for both the rainy and dry seasons: $35 \%$ (IQR 25-57\%) versus 5\% (IQR 49\%).

\section{Setting-specific PFPf}

Urban/rural There were 21 studies conducted in rural areas and 18 in urban areas (as defined by investigators). The overall median PFPf was 38\% (IQR 22-57\%; range $4-81 \%$ ) in rural areas versus $31 \%$ (IQR 19-50\%; range 2$64 \%$ ) in urban settings.

Level of health care 21 studies were conducted in primary care facilities and 18 in the outpatient department of a hospital. The overall median PFPf was 35\% (IQR $22-44 \%$; range $2-81 \%$ ) in primary care settings versus $40 \%$ (IQR 16-56\%; range 4-70\%) in hospitals.

\section{PFPf up to the year 2000 and afterwards}

When comparing PFPf from studies conducted up to the year 2000 (24 studies) with those done afterwards 
Table 1 All 39 included studies showing the Proportions of Fevers associated with Plasmodium falciparum parasitemia (PFPf)

\begin{tabular}{|c|c|c|c|c|c|c|c|c|c|c|}
\hline First author & $\begin{array}{l}\text { Start of the } \\
\text { study }\end{array}$ & Country & $\begin{array}{l}\text { Urban/ } \\
\text { rural area }\end{array}$ & $\begin{array}{l}\text { Place of } \\
\text { recruitment }\end{array}$ & $\begin{array}{l}\text { Clinical inclusion } \\
\text { criteria }\end{array}$ & $\begin{array}{l}\text { Total number of } \\
\text { patients }\end{array}$ & $\begin{array}{l}\text { Overall } \\
\text { PFPf } \\
\end{array}$ & $\begin{array}{l}\text { Age } \\
\text { groups }\end{array}$ & Season & PFPf \\
\hline Rooth [13] & 1986 & Tanzania & Rural & Primary care & History of fever & 596 & $81 \%$ & $\begin{array}{l}\leq 9 \\
\text { years }\end{array}$ & $\begin{array}{l}\text { Both } \\
\text { seasons }\end{array}$ & $81 \%$ \\
\hline $\begin{array}{l}\text { Rougemont } \\
{[22]}\end{array}$ & 1987 & Niger & Rural & Primary care & Temp $\geq 37.5^{\circ} \mathrm{C}$ & 285 & $57 \%$ & $\begin{array}{l}\leq 9 \\
\text { years }\end{array}$ & $\begin{array}{l}\text { Rainy } \\
\text { season }\end{array}$ & $57 \%$ \\
\hline \multirow[t]{3}{*}{ Salako [23] } & 1987 & Nigeria & Urban & Hospital & $\begin{array}{l}\text { History of fever or } \\
\text { elevated temp }\end{array}$ & 7713 & $55 \%$ & $\begin{array}{l}<5 \\
\text { years }\end{array}$ & $\begin{array}{l}\text { Both } \\
\text { seasons }\end{array}$ & $61 \%$ \\
\hline & & & & & & & & $\begin{array}{l}5-18 \\
\text { years }\end{array}$ & & $60 \%$ \\
\hline & & & & & & & & $\begin{array}{l}>18 \\
\text { years }\end{array}$ & & $\overline{41 \%}$ \\
\hline Ejezie [24] & 1988 & Nigeria & Urban & Hospital & $\begin{array}{l}\text { Presumptive } \\
\text { malaria }\end{array}$ & 1188 & $45 \%$ & $\begin{array}{l}\leq 9 \\
\text { years }\end{array}$ & $\begin{array}{l}\text { Both } \\
\text { seasons }\end{array}$ & $45 \%$ \\
\hline \multirow[t]{3}{*}{ Gaye [25] } & 1988 & Senegal & Urban & Primary care & Temp $\geq 38^{\circ} \mathrm{C}$ & 353 & $31 \%$ & $\begin{array}{l}\leq 9 \\
\text { years }\end{array}$ & $\begin{array}{l}\text { Rainy } \\
\text { season }\end{array}$ & $28 \%$ \\
\hline & & & & & & & & $\begin{array}{l}10-14 \\
\text { years } \\
\end{array}$ & & $35 \%$ \\
\hline & & & & & & & & $\begin{array}{l}>14 \\
\text { years } \\
\end{array}$ & & $33 \%$ \\
\hline \multirow[t]{2}{*}{ Olivar [26] } & 1989 & Niger & Urban & Primary care & Temp $>38^{\circ} \mathrm{C}$ & 576 & $35 \%$ & $\begin{array}{l}<5 \\
\text { years }\end{array}$ & $\begin{array}{l}\text { Rainy } \\
\text { season }\end{array}$ & $62 \%$ \\
\hline & & & & & & & & & $\begin{array}{l}\text { Dry } \\
\text { season }\end{array}$ & $5 \%$ \\
\hline Lubanga [27] & 1992 & Uganda & Urban & Hospital & History of fever & 435 & $64 \%$ & $\begin{array}{l}\leq 5 \\
\text { years }\end{array}$ & $\begin{array}{l}\text { Rainy } \\
\text { season }\end{array}$ & $64 \%$ \\
\hline $\begin{array}{l}\text { Meremikwu } \\
{[28]}\end{array}$ & 1993 & Nigeria & Urban & Hospital & Temp $\geq 37.5^{\circ} \mathrm{C}$ & 225 & $63 \%$ & $\begin{array}{l}<5 \\
\text { years }\end{array}$ & $\begin{array}{l}\text { Rainy } \\
\text { season }\end{array}$ & $63 \%$ \\
\hline Redd [29] & 1993 & Malawi & Rural & Hospital & $\begin{array}{l}\text { Presumptive } \\
\text { malaria }\end{array}$ & 1124 & $60 \%$ & $\begin{array}{l}<5 \\
\text { years }\end{array}$ & $\begin{array}{l}\text { Rainy } \\
\text { season }\end{array}$ & $60 \%$ \\
\hline Olaleye [30] & 1993 & $\begin{array}{l}\text { the } \\
\text { Gambia }\end{array}$ & Rural & Primary care & $\begin{array}{l}\text { History of fever or } \\
\text { temp } \geq 37.5^{\circ} \mathrm{C}\end{array}$ & 407 & $59 \%$ & $\begin{array}{l}\leq 9 \\
\text { years }\end{array}$ & $\begin{array}{l}\text { Rainy } \\
\text { season }\end{array}$ & $59 \%$ \\
\hline \multirow[t]{2}{*}{ Weber [31] } & 1993 & $\begin{array}{l}\text { the } \\
\text { Gambia }\end{array}$ & Urban & Hospital & $\begin{array}{l}\text { Presumptive } \\
\text { malaria }\end{array}$ & 440 & $7 \%$ & $\begin{array}{l}<5 \\
\text { years }\end{array}$ & $\begin{array}{l}\text { Rainy } \\
\text { season }\end{array}$ & $17 \%$ \\
\hline & & & & & & & & & $\begin{array}{l}\text { Dry } \\
\text { season }\end{array}$ & $4 \%$ \\
\hline \multirow[t]{4}{*}{ Gaye [32] } & 1994 & Senegal & Urban & Hospital & History of fever & 762 & $31 \%$ & All ages & $\begin{array}{l}\text { Rainy } \\
\text { season }\end{array}$ & $59 \%$ \\
\hline & & & & & & & & & $\begin{array}{l}\text { Dry } \\
\text { season }\end{array}$ & $5 \%$ \\
\hline & & & & & & & & $\begin{array}{l}<5 \\
\text { years } \\
\end{array}$ & $\begin{array}{l}\text { Both } \\
\text { seasons }\end{array}$ & $14 \%$ \\
\hline & & & & & & & & $\begin{array}{l}\geq 5 \\
\text { years }\end{array}$ & & $36 \%$ \\
\hline \multirow[t]{2}{*}{ Font [14] } & 1995 & Tanzania & Rural & Primary care & $\begin{array}{l}\text { History of fever or } \\
\text { temp }>37.5^{\circ} \mathrm{C}\end{array}$ & 641 & $44 \%$ & $\begin{array}{l}<5 \\
\text { years }\end{array}$ & $\begin{array}{l}\text { Rainy } \\
\text { season }\end{array}$ & $57 \%$ \\
\hline & & & & & & & & $\begin{array}{l}\geq 5 \\
\text { years }\end{array}$ & & $24 \%$ \\
\hline Tarimo [33] & 1996 & Tanzania & Urban & Hospital & $\begin{array}{l}\text { Presumptive } \\
\text { malaria }\end{array}$ & 400 & $52 \%$ & All ages & $\begin{array}{l}\text { Both } \\
\text { seasons }\end{array}$ & $52 \%$ \\
\hline Cooke [34] & 1996 & $\begin{array}{l}\text { the } \\
\text { Gambia }\end{array}$ & Rural & Hospital & $\begin{array}{l}\text { History of fever or } \\
\text { elevated temp }\end{array}$ & 398 & $36 \%$ & All ages & $\begin{array}{l}\text { Rainy } \\
\text { season }\end{array}$ & $36 \%$ \\
\hline \multirow[t]{2}{*}{ Muhe [35] } & 1996 & Ethiopia & Rural & Primary care & $\begin{array}{l}\text { History of fever or } \\
\text { temp } \geq 38^{\circ} \mathrm{C}\end{array}$ & 2490 & $22 \%$ & $\begin{array}{l}<5 \\
\text { years }\end{array}$ & $\begin{array}{l}\text { Rainy } \\
\text { season }\end{array}$ & $30 \%$ \\
\hline & & & & & & & & & $\begin{array}{l}\text { Dry } \\
\text { season }\end{array}$ & $6 \%$ \\
\hline
\end{tabular}


Table 1 All 39 included studies showing the Proportions of Fevers associated with Plasmodium falciparum parasitemia (PFPf) (Continued)

\begin{tabular}{|c|c|c|c|c|c|c|c|c|c|c|}
\hline Cortes [36] & 1996 & Mauritania & Urban & Hospital & History of fever & 416 & $19 \%$ & All ages & $\begin{array}{l}\text { Both } \\
\text { seasons }\end{array}$ & $19 \%$ \\
\hline \multirow[t]{2}{*}{ Oster [37] } & 1996 & Tanzania & Rural & Hospital & $\begin{array}{l}\text { History of fever or } \\
\text { temp } \geq 37.5^{\circ} \mathrm{C}\end{array}$ & 168 & $14 \%$ & Adults & $\begin{array}{l}\text { Rainy } \\
\text { season }\end{array}$ & $20 \%$ \\
\hline & & & & & & & & & $\begin{array}{l}\text { Dry } \\
\text { season }\end{array}$ & $0 \%$ \\
\hline Nsimba [15] & 1997 & Tanzania & Rural & Primary care & $\begin{array}{l}\text { Presumptive } \\
\text { malaria }\end{array}$ & 449 & $38 \%$ & $\begin{array}{l}\leq 5 \\
\text { years }\end{array}$ & $\begin{array}{l}\text { Rainy } \\
\text { season }\end{array}$ & $38 \%$ \\
\hline \multirow[t]{3}{*}{ Akim [38] } & 1997 & Tanzania & Rural & Primary care & History of fever & 6580 & $43 \%$ & $\begin{array}{l}\leq 6 \\
\text { years }\end{array}$ & $\begin{array}{l}\text { Both } \\
\text { seasons }\end{array}$ & $57 \%$ \\
\hline & & & & & & & & $\begin{array}{l}7-15 \\
\text { years }\end{array}$ & & $44 \%$ \\
\hline & & & & & & & & $\begin{array}{l}>15 \\
\text { years }\end{array}$ & & $27 \%$ \\
\hline \multirow[t]{3}{*}{ Arness [39] } & 1998 & Kenya & Rural & Primary care & $\begin{array}{l}\text { Presumptive } \\
\text { malaria }\end{array}$ & 2796 & $29 \%$ & $\begin{array}{l}<5 \\
\text { years }\end{array}$ & $\begin{array}{l}\text { Both } \\
\text { seasons }\end{array}$ & $32 \%$ \\
\hline & & & & & & & & $\begin{array}{l}5-15 \\
\text { years }\end{array}$ & & $35 \%$ \\
\hline & & & & & & & & $\begin{array}{l}>15 \\
\text { years }\end{array}$ & & $23 \%$ \\
\hline Tarimo [40] & 2000 & Tanzania & Rural & Hospital & $\begin{array}{l}\text { Malaria based on } \\
\text { IMCl criteria }\end{array}$ & 395 & $70 \%$ & $\begin{array}{l}\leq 5 \\
\text { years }\end{array}$ & $\begin{array}{l}\text { Rainy } \\
\text { season }\end{array}$ & $70 \%$ \\
\hline \multirow[t]{2}{*}{$\begin{array}{l}\text { Guthmann } \\
\text { [41] }\end{array}$} & 2000 & Uganda & Rural & Hospital & $\begin{array}{l}\text { Presumptive } \\
\text { malaria }\end{array}$ & 742 & $57 \%$ & $\begin{array}{l}<5 \\
\text { years }\end{array}$ & $\begin{array}{l}\text { Rainy } \\
\text { season }\end{array}$ & $64 \%$ \\
\hline & & & & & & & & $\begin{array}{l}>5 \\
\text { years }\end{array}$ & & $52 \%$ \\
\hline \multirow[t]{3}{*}{ Gouagna [42] } & 2000 & Kenya & Rural & Primary care & $\begin{array}{l}\text { Presumptive } \\
\text { malaria }\end{array}$ & 3754 & $47 \%$ & $\begin{array}{l}<5 \\
\text { years }\end{array}$ & $\begin{array}{l}\text { Both } \\
\text { seasons }\end{array}$ & $55 \%$ \\
\hline & & & & & & & & $\begin{array}{l}5-15 \\
\text { years }\end{array}$ & & $57 \%$ \\
\hline & & & & & & & & $\begin{array}{l}>15 \\
\text { years }\end{array}$ & & $36 \%$ \\
\hline \multirow[t]{2}{*}{$\begin{array}{l}\text { Raharimalala } \\
\text { [43] }\end{array}$} & 2001 & $\begin{array}{l}\text { Madagas- } \\
\text { car }\end{array}$ & Rural & Primary care & $\begin{array}{l}\text { Presumptive } \\
\text { malaria }\end{array}$ & 149 & $35 \%$ & $\begin{array}{l}<15 \\
\text { years }\end{array}$ & $\begin{array}{l}\text { Rainy } \\
\text { season }\end{array}$ & $37 \%$ \\
\hline & & & & & & & & $\begin{array}{l}\geq 15 \\
\text { years }\end{array}$ & & $29 \%$ \\
\hline \multirow[t]{2}{*}{$\begin{array}{l}\text { Assoumou } \\
{[44]}\end{array}$} & 2001 & $\begin{array}{l}\text { Ivory } \\
\text { Coast }\end{array}$ & Urban & Hospital & Temp $\geq 37.5^{\circ} \mathrm{C}$ & 902 & $29 \%$ & $\begin{array}{l}<5 \\
\text { years }\end{array}$ & $\begin{array}{l}\text { Both } \\
\text { seasons }\end{array}$ & $31 \%$ \\
\hline & & & & & & & & $\begin{array}{l}5-15 \\
\text { years }\end{array}$ & & $27 \%$ \\
\hline \multirow[t]{5}{*}{ Othnigue [45] } & 2002 & Chad & Urban & Primary care & $\begin{array}{l}\text { Presumptive } \\
\text { malaria }\end{array}$ & 712 & $30 \%$ & All ages & $\begin{array}{l}\text { Rainy } \\
\text { season }\end{array}$ & $35 \%$ \\
\hline & & & & & & & & & $\begin{array}{l}\text { Dry } \\
\text { season }\end{array}$ & $12 \%$ \\
\hline & & & & & & & & $\begin{array}{l}<5 \\
\text { years }\end{array}$ & $\begin{array}{l}\text { Both } \\
\text { seasons }\end{array}$ & $19 \%$ \\
\hline & & & & & & & & $\begin{array}{l}5-14 \\
\text { years }\end{array}$ & & $50 \%$ \\
\hline & & & & & & & & $\begin{array}{l}\geq 15 \\
\text { years }\end{array}$ & & $27 \%$ \\
\hline Zurovac [46] & 2002 & Kenya & Rural & Primary care & $\begin{array}{l}\text { Sent for malaria } \\
\text { test by clinician in } \\
\text { charge }\end{array}$ & 261 & $13 \%$ & $\begin{array}{l}\geq 5 \\
\text { years }\end{array}$ & $\begin{array}{l}\text { Rainy } \\
\text { season }\end{array}$ & $13 \%$ \\
\hline Malik [47] & 2002 & Sudan & Urban & Hospital & History of fever & 655 & $12 \%$ & $\begin{array}{l}<5 \\
\text { years }\end{array}$ & $\begin{array}{l}\text { Dry } \\
\text { season }\end{array}$ & $11 \%$ \\
\hline
\end{tabular}


Table 1 All 39 included studies showing the Proportions of Fevers associated with Plasmodium falciparum parasitemia (PFPf) (Continued)

\begin{tabular}{|c|c|c|c|c|c|c|c|c|c|c|}
\hline & & & & & & & & $\begin{array}{l}5-16 \\
\text { years }\end{array}$ & & $13 \%$ \\
\hline \multirow[t]{3}{*}{ Wang [48] } & 2002 & $\begin{array}{l}\text { Ivory } \\
\text { coast }\end{array}$ & Urban & Primary care & $\begin{array}{l}\text { History of fever or } \\
\text { temp } \geq 37.5^{\circ} \mathrm{C}\end{array}$ & 429 & $35 \%$ & $\begin{array}{l}\leq 5 \\
\text { years }\end{array}$ & $\begin{array}{l}\text { Rainy } \\
\text { season }\end{array}$ & $36 \%$ \\
\hline & & & & & & & & $\begin{array}{l}6-15 \\
\text { years }\end{array}$ & & $44 \%$ \\
\hline & & & & & & & & $\begin{array}{l}>15 \\
\text { years }\end{array}$ & & $26 \%$ \\
\hline \multirow[t]{3}{*}{ Wang [49] } & 2002 & Burkina & Urban & Primary care & $\begin{array}{l}\text { History of fever or } \\
\text { temp } \geq 37.5^{\circ} \mathrm{C}\end{array}$ & 560 & $22 \%$ & $\begin{array}{l}\leq 5 \\
\text { years }\end{array}$ & $\begin{array}{l}\text { Dry } \\
\text { season }\end{array}$ & $22 \%$ \\
\hline & & & & & & & & $\begin{array}{l}6-15 \\
\text { years }\end{array}$ & & $37 \%$ \\
\hline & & & & & & & & $\begin{array}{l}>15 \\
\text { years }\end{array}$ & & $18 \%$ \\
\hline \multirow[t]{3}{*}{ Wang [50] } & 2003 & Tanzania & Urban & Primary care & $\begin{array}{l}\text { History of fever or } \\
\text { temp } \geq 37.5^{\circ} \mathrm{C}\end{array}$ & 717 & $5 \%$ & $\begin{array}{l}\leq 5 \\
\text { years }\end{array}$ & $\begin{array}{l}\text { Dry } \\
\text { season }\end{array}$ & $5 \%$ \\
\hline & & & & & & & & $\begin{array}{l}6-15 \\
\text { years }\end{array}$ & & $7 \%$ \\
\hline & & & & & & & & $\begin{array}{l}>15 \\
\text { years }\end{array}$ & & $4 \%$ \\
\hline \multirow[t]{3}{*}{ Wang [51] } & 2003 & Benin & Urban & Primary care & $\begin{array}{l}\text { History of fever or } \\
\text { temp } \geq 37.5^{\circ} \mathrm{C}\end{array}$ & 379 & $2 \%$ & $\begin{array}{l}\leq 5 \\
\text { years }\end{array}$ & $\begin{array}{l}\text { Dry } \\
\text { season }\end{array}$ & $4 \%$ \\
\hline & & & & & & & & $\begin{array}{l}6-15 \\
\text { years }\end{array}$ & & $0 \%$ \\
\hline & & & & & & & & $\begin{array}{l}>15 \\
\text { years }\end{array}$ & & $1 \%$ \\
\hline Yacoub [52] & 2003 & Zanzibar & Rural & Primary care & $\begin{array}{l}\text { History of fever } \\
\text { or temp }>37.5^{\circ} \mathrm{C}\end{array}$ & 207 & $77 \%$ & $\begin{array}{l}\leq 5 \\
\text { years }\end{array}$ & $\begin{array}{l}\text { Rainy } \\
\text { season }\end{array}$ & $77 \%$ \\
\hline \multirow[t]{2}{*}{$\begin{array}{l}\text { Ogungbami- } \\
\text { gbe [53] }\end{array}$} & 2004 & Nigeria & Urban & Hospital & Temp $\geq 37.5^{\circ} \mathrm{C}$ & 646 & $53 \%$ & $\begin{array}{l}\leq 9 \\
\text { years }\end{array}$ & $\begin{array}{l}\text { Rainy } \\
\text { season }\end{array}$ & $62 \%$ \\
\hline & & & & & & & & & $\begin{array}{l}\text { Dry } \\
\text { season }\end{array}$ & $28 \%$ \\
\hline \multirow[t]{2}{*}{ Kachur [54] } & 2004 & Tanzania & Rural & Primary care & History of fever & 769 & $31 \%$ & $\begin{array}{l}<5 \\
\text { years }\end{array}$ & $\begin{array}{l}\text { Rainy } \\
\text { season }\end{array}$ & $43 \%$ \\
\hline & & & & & & & & $\begin{array}{l}\geq 5 \\
\text { years }\end{array}$ & & $23 \%$ \\
\hline \multirow[t]{3}{*}{ Reyburn [16] } & 2005 & Tanzania & Rural & Hospital & $\begin{array}{l}\text { Sent for malaria } \\
\text { test by clinician in } \\
\text { charge }\end{array}$ & 2397 & $15 \%$ & $\begin{array}{l}<5 \\
\text { years }\end{array}$ & $\begin{array}{l}\text { Rainy } \\
\text { season }\end{array}$ & $21 \%$ \\
\hline & & & & & & & & $\begin{array}{l}5-15 \\
\text { years } \\
\end{array}$ & & $17 \%$ \\
\hline & & & & & & & & $\begin{array}{l}>15 \\
\text { years }\end{array}$ & & $8 \%$ \\
\hline Reyburn [55] & 2005 & Tanzania & Rural & Hospital & $\begin{array}{l}\text { Sent for malaria } \\
\text { test by clinician in } \\
\text { charge }\end{array}$ & 214 & $4 \%$ & All ages & $\begin{array}{l}\text { Rainy } \\
\text { season }\end{array}$ & $4 \%$ \\
\hline Mens [56] & 2007 & Kenya & Rural & Primary care & $\begin{array}{l}\text { History of fever or } \\
\text { temp } \geq 37.5^{\circ} \mathrm{C}\end{array}$ & 650 & $17 \%$ & $\begin{array}{l}\leq 12 \\
\text { years }\end{array}$ & $\begin{array}{l}\text { Rainy } \\
\text { season }\end{array}$ & $17 \%$ \\
\hline
\end{tabular}

(15 studies), there was a clear reduction in the median PFPf from 44\% (IQR 31-58\%; range 7-81\%) to 22\% (IQR 13-33\%; range 2-77\%) (Figure 3). This dramatic decline is likely to reflect a true change since all variables listed above and which were shown to have an effect on PFPf were well balanced between the two groups of studies.
For studies before and after 2000 respectively, 17.5/24 (73\%) and 9.5/15 (63\%) studies were conducted during the rainy season (when a study took place during both the rainy and the dry season, the count was 0.5 study for each of the season) ( $\mathrm{p}=0.45) ; 14 / 24(58 \%)$ and $7 / 15$ $(47 \%)$ studies were conducted in a rural setting 


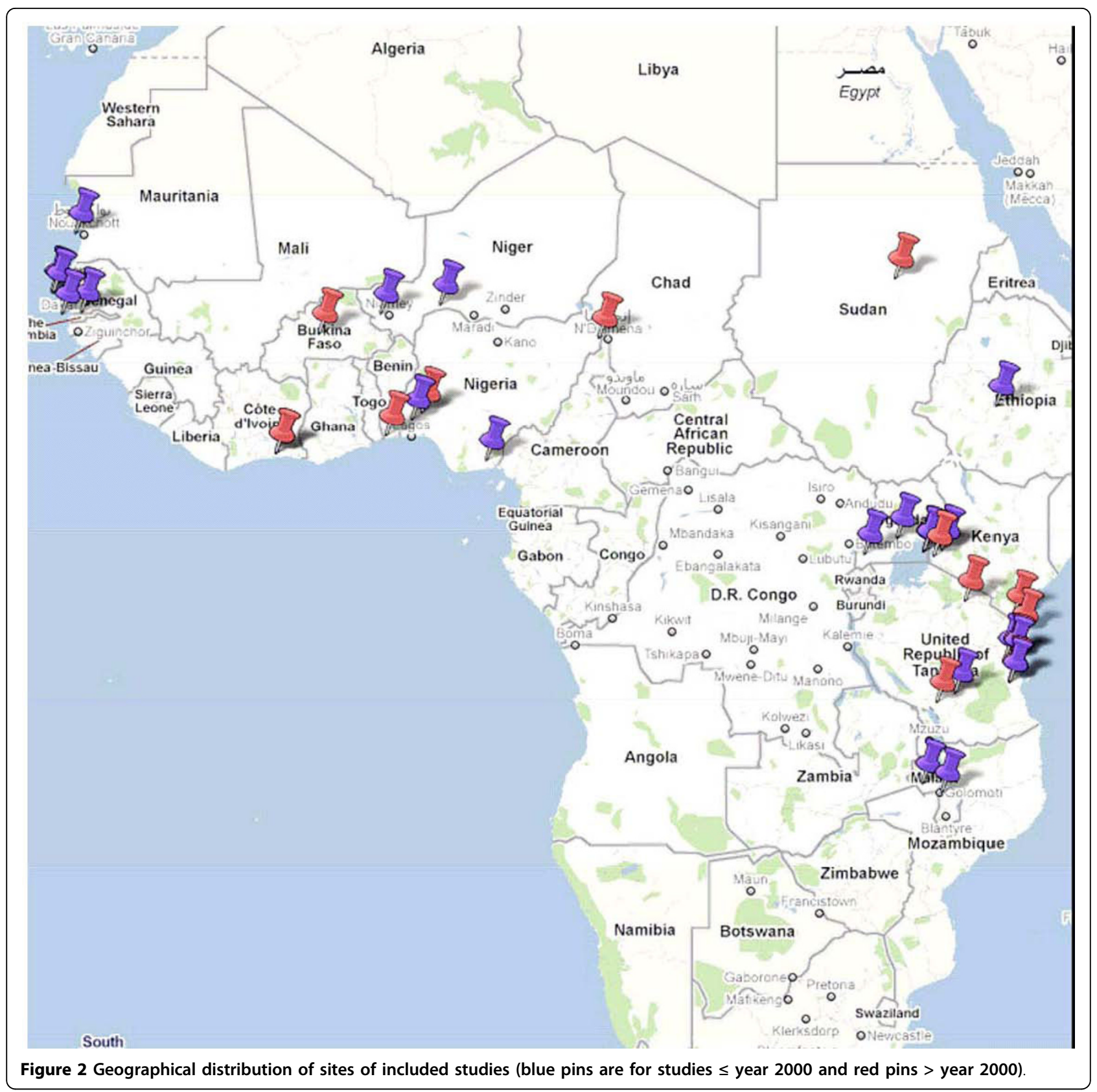

$(\mathrm{p}=0.53)$; and $11 / 24(46 \%)$ and $10 / 15(67 \%)$ were conducted at primary care level $(\mathrm{p}=0.32)$.

In the 11 studies from Tanzania (considered mostly as a highly endemic area), PFPf in children under five years during the rainy season decreased from $81 \%$ in 1986-88 [13] to $57 \%$ in 1995 [14], $38 \%$ in 1997 [15] and 21\% in 2005 [16].

To further check for potential confounding factors, a stratified analysis was conducted including each of the variables listed above. The reduction of PFPf over time was confirmed since median PFPfs were almost always lower for the years after 2000 compared to the years $\leq 2000$ (see Figure 3). There was one exception in the case of the data collected during the dry season.

\section{Discussion}

This systematic review demonstrates a $50 \%$ reduction of PFPf for the period after year 2000 when compared to that of $\leq 2000$ ( $22 \%$ versus $44 \%$ ). This decrease by half of the proportion of malaria cases among fever episodes is likely to be due to a reduction of malaria transmission. It mirrors the reduction observed in parasite prevalence rates collected from community cross-sectional surveys in sub-Saharan Africa during the same time periods 


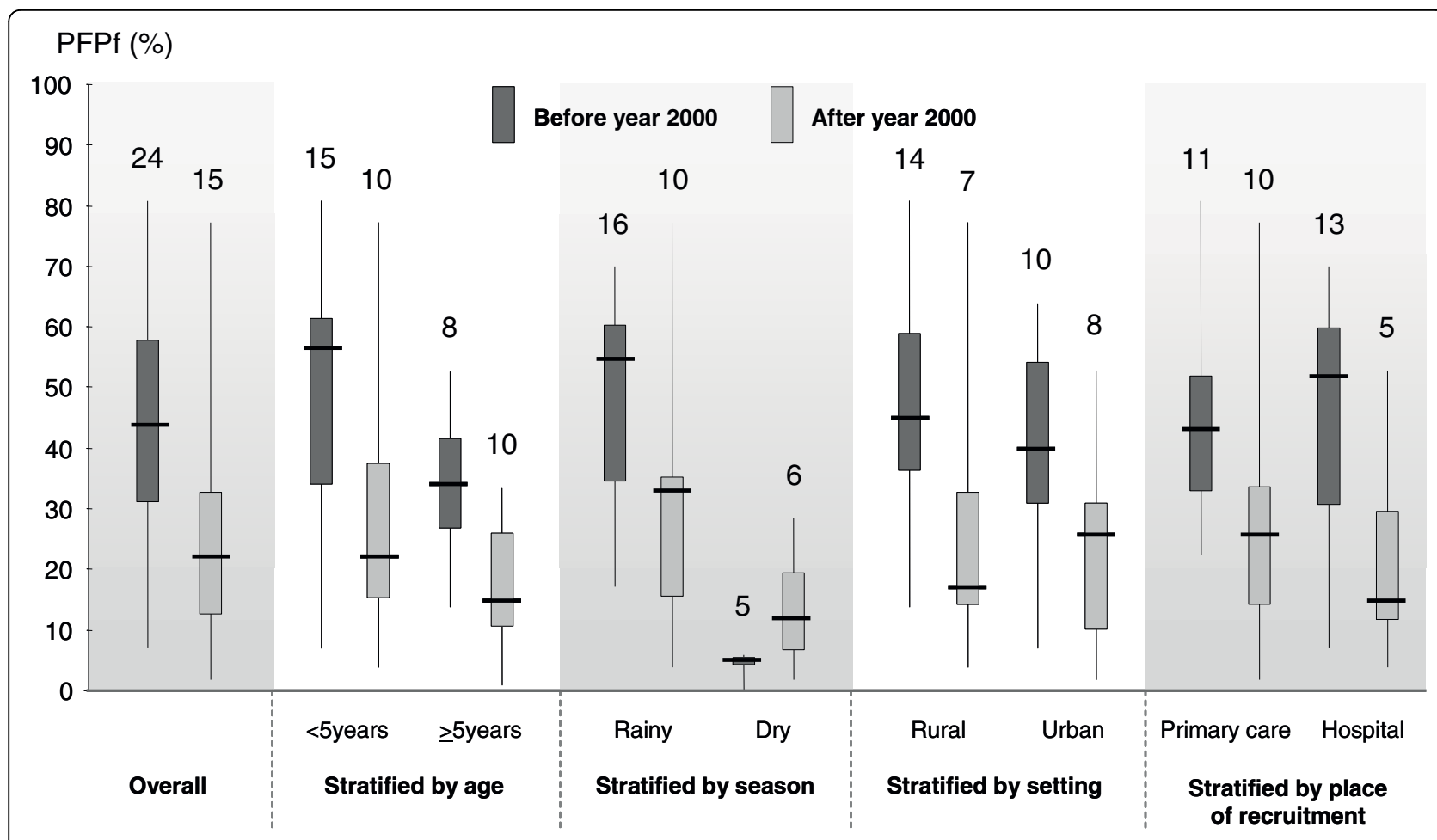

Figure 3 Comparison between the Proportions of Fevers associated with Plasmodium falciparum parasitemia (PFPf) in years $\leq 2000$ and $>2000$, stratified by baseline characteristics (numbers above plots corresponds to the number of studies involved).

(17\% after year 2000 versus $37 \%$ before) [6]. A decrease in malaria is now observed in many settings across subSaharan Africa, mainly because of the large scale implementation of effective control measures following a drastic increase of funding since the year 2000 [12]. Recently, Okiro \& Snow demonstrated the clear linear relationship between the risk of infection among febrile children and parasite prevalence in the community [17].

An inherent difficulty with systematic reviews is that they look at studies which may not be comparable. In the present review, the most recent studies report a lower proportion of malaria attributable fevers, but some of these later studies have been conducted in areas of lower endemicity than those performed previously. This is especially true for some studies in Tanzania, a country largely represented in this systematic review. In part, this was due to the fact that some of the recent studies were conducted in urban and periurban settings, whereas old ones were traditionally done in rural places, where transmission of malaria is usually higher. Part of the reduction in the proportion of malaria among fevers may thus be ascribed to selection biases. To address this problem, stratified analyses for variables known to have an effect on PFPf were performed. These analyses confirmed the reduction of PFPf over time when controlling for these different factors. The only exception was for dry season and this is easily explainable by the fact that the median PFPf was always rather low, irrespective of the period. When data are available, such as in Tanzania for example, the substantial reduction of PFPf parallels closely the decline of incidence of malaria episodes and overall mortality in children under five observed in the same areas over the last fifteen years [18,19] (Khatib et al, unpublished data).

Although the reduction of PFPf was considerable, it is unclear how representative these data are at this point in time. Data are over-represented in sites with research institutions or special situations and it would be a great importance to confirm such trends in other settings, especially in the largest malarious countries on the continent, the Democratic Republic of the Congo and Nigeria. Also, within a country or region, the reductions are not necessarily uniform. There are still a number of places that harbour PFPf over $50 \%$, especially so in areas where healthcare is not readily accessible.

On the other hand, there is no doubt that malaria control is very successful in many countries [5] and these trends are expected as a result of greatly improved preventive activities. In any case, the immediate practical implication of the changing epidemiology of fever episodes is the much increased need for a systematic laboratory diagnosis of every case before initiating treatment for malaria [20]. 


\section{Conclusions}

This systematic review demonstrates a considerable reduction of PFPf in sub-Saharan Africa between the periods before the year 2000 and from 2001 onwards (44\% versus $22 \%$ ). With only around a fifth of all fever episodes being associated with malaria parasitaemia, this review provides strong evidence to support the new WHO policy of laboratory-based diagnosis and treatment upon result [21]. This should insure appropriate care of non-malaria fevers and rationale use of antimalarials. The relative cost effectiveness and value of introducing diagnosis for febrile children will obviously depend on infection prevalence in the community.

\section{Financial disclosure}

VdA was supported by a grant of the Swiss National Science Foundation (Grant \# 3270B0-109696). CL and BG have permanent positions in their own institution. The funder had no role in study design, data collection and analysis, decision to publish, or preparation of the manuscript.

\section{Acknowledgements}

The authors are grateful to Piero Olliaro for critical reading.

\section{Author details}

${ }^{1}$ Swiss Tropical and Public Health Institute, P.O. Box, 4002 Basel, Switzerland.

${ }^{2}$ University of Basel, Basel, Switzerland. ${ }^{3}$ Department of Ambulatory Care and Community Medicine, Infectious Disease service, University Hospital, Lausanne, Switzerland.

\section{Authors' contributions}

VdA did the literature search, the analyses, and wrote the first draft of the manuscript. BG counterchecked the selection of articles and contributed to the manuscript writing. $C L$ contributed to the manuscript. All authors read and approved the final manuscript.

\section{Competing interests}

The authors declare that they have no competing interests.

Received: 11 May 2010 Accepted: 22 August 2010

Published: 22 August 2010

\section{References}

1. Roll Back Malaria: The global malaria action plan. For a malaria-free world. Geneva, Switzerland 2008.

2. Rugemalila JB, Wanga CL, Kilama WL: Sixth Africa Malaria Day in 2006: how far have we come after the Abuja Declaration? Malar J 2006, 5:102.

3. Roll Back Malaria: World malaria report 2005. [http://www.rollbackmalaria. org/wmr2005], (last accessed 16.08.2010).

4. O'Meara WP, Bejon P, Mwangi TW, Okiro EA, Peshu N, Snow RW, Newton CRJC, Marsh K: Effect of a fall in malaria transmission on morbidity and mortality in Kilifi, Kenya. Lancet 2008, 372:1555-1562.

5. World Health Organization: World malaria report 2009.[http://www.who. int/malaria/world_malaria_report_2009/en/index.html], (last accessed 16.08.2010).

6. Guerra CA, Gikandi PW, Tatem AJ, Noor AM, Smith DL, Hay SI, Snow RW: The limits and intensity of Plasmodium falciparum transmission: implications for malaria control and elimination worldwide. PLOS Med 2008, 5:e38.

7. De Savigny D: Chair's opening remarks to the Roll Back Malaria Working Group on Scalable Malaria Vector Control Working Group Meeting , 1-10-2007.
8. Snow RW, Eckert E, Teklehaimanot A: Estimating the needs for artesunatebased combination therapy for malaria case-management in Africa. Trends Parasitol 2003, 19:363-369.

9. Chima Rl, Goodman CA, Mills A: The economic impact of malaria in Africa: a critical review of the evidence. Health Policy 2003, 63:17-36.

10. Rowe AK, Rowe SY, Snow RW, Korenromp EL, Schellenberg JRA, Stein C, Nahlen BL, Bryce J, Black RE, Steketee RW: The burden of malaria mortality among African children in the year 2000. Int J Epidemiol 2006, 35:691-704.

11. Central Intelligence Agency: The World Factbook.[https://www.cia.gov/ library/publications/the-world-factbook/index.html], (last accessed 16.08.2010)

12. Waddington C: Does earmarked donor funding make it more or less likely that developing countries will allocate their resources towards programmes that yield the greatest health benefits? Bull World Health Organ 2004, 82:703-706, discussion 706-708.

13. Rooth I, Björkman A: Fever episodes in a holoendemic malaria area of Tanzania: parasitological and clinical findings and diagnostic aspects related to malaria. Trans R Soc Trop Med Hyg 1992, 86:479-482.

14. Font F, Alonso González M, Nathan R, Kimario J, Lwilla F, Ascaso C, Tanner M, Menéndez C, Alonso PL: Diagnostic accuracy and case management of clinical malaria in the primary health services of a rural area in south-eastern Tanzania. Trop Med Int Health 2001, 6:423-428.

15. Nsimba SED, Massele AY, Eriksen J, Gustafsson LL, Tomson G, Warsame M: Case management of malaria in under-fives at primary health care facilities in a Tanzanian district. Trop Med Int Health 2002, 7:201-209.

16. Reyburn H, Mbakilwa H, Mwangi R, Mwerinde O, Olomi R, Drakeley C, Whitty CJM: Rapid diagnostic tests compared with malaria microscopy for guiding outpatient treatment of febrile illness in Tanzania: randomised trial. BMJ 2007, 334:403.

17. Okiro EA, Snow RW: The relationship between reported fever and Plasmodium falciparum infection in African children. Malar J 2010, 9:99.

18. Schellenberg D, Menendez C, Aponte J, Guinovart C, Mshinda H, Tanner M, Alonso $P$ : The changing epidemiology of malaria in Ifakara Town, southern Tanzania. Trop Med Int Health 2004, 9:68-76.

19. Masanja H, de Savigny D, Smithson P, Schellenberg J, John T, Mbuya C, Upunda G, Boerma T, Victora C, Smith T, Mshinda H: Child survival gains in Tanzania: analysis of data from demographic and health surveys. Lancet 2008, 371:1276-1283.

20. D'Acremont V, Lengeler C, Mshinda H, Mtasiwa D, Tanner M, Genton B: Time to move from presumptive malaria treatment to laboratoryconfirmed diagnosis and treatment in African children with fever. PLOS Med 2009, 6:e252.

21. World Health Organization: Guidelines for the treatment of malaria, second edition. Geneva, Switzerland 2010.

22. Rougemont A, Breslow N, Brenner E, Moret AL, Dumbo O, Dolo A, Soula G, Perrin L: Epidemiological basis for clinical diagnosis of childhood malaria in endemic zone in West Africa. Lancet 1991, 338:1292-1295.

23. Salako LA, Ajayi FO, Sowunmi A, Walker O: Malaria in Nigeria: a revisit. Ann Trop Med Parasitol 1990, 84:435-445.

24. Ejezie GC, Ezedinachi EN: Malaria parasite density and body temperature in children under 10 years of age in Calabar, Nigeria. Trop Geogr Med 1992, 44:97-101.

25. Gaye O, Bah IB, Bengue E, Diallo S, Faye O: Malaria morbidity in the urban environment. Study of 353 fever attacks. Med Trop 1989, 49:401-404.

26. Olivar M, Develoux M, Chegou Abari A, Loutan L: Presumptive diagnosis of malaria results in a significant risk of mistreatment of children in urban Sahel. Trans R Soc Trop Med Hyg 1991, 85:729-730.

27. Lubanga RG, Norman S, Ewbank D, Karamagi C: Maternal diagnosis and treatment of children's fever in an endemic malaria zone of Uganda: implications for the malaria control programme. Acta Trop 1997, 68:53-64.

28. Meremikwu MM, Asindi AA, Ezedinachi EN, Imananagha L, Etim B, OyoIta A, Tomkins M: Hyperventilation in febrile Nigerian children without pneumonia: an evaluation of the influence of anaemia. Cent Afr J Med 1995, 41:248-252.

29. Redd SC, Kazembe PN, Luby SP, Nwanyanwu O, Hightower AW, Ziba C, Wirima JJ, Chitsulo L, Franco C, Olivar M: Clinical algorithm for treatment of Plasmodium falciparum malaria in children. Lancet 1996, 347:223-227.

30. Olaleye BO, Williams LA, D'Alessandro U, Weber MM, Mulholland K, Okorie C, Langerock P, Bennet S, Greenwood BM: Clinical predictors of malaria in Gambian children with fever or a history of fever. Trans $R$ Soc Trop Med Hyg 1998, 92:300-304. 
31. Weber MW, Mulholland EK, Jaffar S, Troedsson H, Gove S, Greenwood BM: Evaluation of an algorithm for the integrated management of childhood illness in an area with seasonal malaria in the Gambia. Bull World Health Organ 1997, 75(Suppl 1):25-32.

32. Gaye O, Faye O, Ndir O, Feller-Dansokho E, Faye O, Dieng Y, Lakh NC, Diallo S: Malaria in an urban environment: the case of the city of Rufisque in Senegal. Dakar Med 1997, 42:54-58.

33. Tarimo DS, Mpembeni R, Kawawa H, Mshana TC: Appraisal of the acridine orange method for rapid malaria diagnosis at three Tanzanian district hospitals. East Afr Med J 1998, 75:504-507.

34. Cooke AH, Chiodini PL, Doherty T, Moody AH, Ries J, Pinder M: Comparison of a parasite lactate dehydrogenase-based immunochromatographic antigen detection assay (OptiMAL) with microscopy for the detection of malaria parasites in human blood samples. Am J Trop Med Hyg 1999, 60:173-176.

35. Muhe L, Oljira B, Degefu H, Enquesellassie F, Weber MW: Clinical algorithm for malaria during low and high transmission seasons. Arch Dis Child 81:216-220.

36. Cortes H, Morillas-Márquez F, Valero A: Malaria in Mauritania: the first cases of malaria endemic to Nouakchott. Trop Med Int Health 2003, 8:297-300.

37. Oster N, Krause E, Hatz C: Towards a rational malaria management at district hospital level: exploratory case series of febrile adult patients in a holoendemic area of Tanzania. Trop Doct 2000, 30:203-207.

38. Akim NI, Drakeley C, Kingo T, Simon B, Senkoro K, Sauerwein RW: Dynamics of $P$. falciparum gametocytemia in symptomatic patients in an area of intense perennial transmission in Tanzania. Am J Trop Med Hyg 2000, 63:199-203.

39. Arness MK, Bradshaw RD, Biomndo K, Shanks GD: Epidemiology of highland malaria in western Kenya. East Afr Med J 2003, 80:253-259.

40. Tarimo DS, Minjas JN, Bygbjerg IC: Malaria diagnosis and treatment under the strategy of the integrated management of childhood illness (IMCI): relevance of laboratory support from the rapid immunochromatographic tests of ICT Malaria P.f/P.v and OptiMal. Ann Trop Med Parasitol 2001, 95:437-444.

41. Guthmann JP, Ruiz A, Priotto G, Kiguli J, Bonte L, Legros D: Validity, reliability and ease of use in the field of five rapid tests for the diagnosis of Plasmodium falciparum malaria in Uganda. Trans $R$ Soc Trop Med Hyg 2002, 96:254-257.

42. Gouagna LC, Okech BA, Kabiru EW, Killeen GF, Obare P, Ombonya S, Bier JC, Knols BGJ, Githure JI, Yan G: Infectivity of Plasmodium falciparum gametocytes in patients attending rural health centres in western Kenya. East Afr Med J 2003, 80:627-634.

43. Raharimalala LA, Rabarijaona L, Randrianarivelojosia M, Razanavololo F, Rason MA, Andrianantenaina HB, Andrianaivolambo L, Rakotoniaina JC, Leong Pock Tsi JM, Rajaonarivelo E, Léon T, Duchemin JB, Ariey F: Malaria study in the cyclone risk zone: entomological, diagnostic and therapeutic approach in the southeastern region of Madagascar. Arch Inst Pasteur Madagascar 2002, 68:79-85.

44. Assoumou A, Adoubryn KD, Aboum KS, Kouadio-Yapo CG, Ouhon J: Symptomatic and asymptomatic Plasmodium falciparum infection in children from 6 months to 6 years old in the Abobo general hospital (Abidjan, Côte d'Ivoire). Bull Soc Pathol Exot 2008, 101:50-53.

45. Othnigué N, Wyss K, Tanner M, Genton B: Urban malaria in the Sahel: prevalence and seasonality of presumptive malaria and parasitaemia at primary care level in Chad. Trop Med Int Health 2006, 11:204-210.

46. Zurovac D, Midia B, Ochola SA, English M, Snow RW: Microscopy and outpatient malaria case management among older children and adults in Kenya. Trop Med Int Health 2006, 11:432-440.

47. Malik EM, Eltahir HG, Ahmed ES: Clinical and laboratory aspects of malaria among children with fever in a low transmission area of Sudan. East Mediterr Health J 2005, 11:753-761.

48. Wang S, Lengeler C, Smith TA, Vounatsou P, Cissé G, Tanner M: Rapid Urban Malaria Appraisal (RUMA) III: epidemiology of urban malaria in the municipality of Yopougon (Abidjan). Malar J 2006, 5:29.

49. Wang S, Lengeler C, Smith TA, Vounatsou P, Diadie DA, Pritroipa X, Convelbo N, Kientga M, Tanner M: Rapid urban malaria appraisal (RUMA) I: epidemiology of urban malaria in Ouagadougou. Malar J 2005, 4:43.

50. Wang S, Lengeler C, Mtasiwa D, Mshana T, Manane L, Maro G, Tanner M: Rapid Urban Malaria Appraisal (RUMA) II: epidemiology of urban malaria in Dar es Salaam (Tanzania). Malar J 2006, 5:28.
51. Wang S, Lengeler C, Smith TA, Vounatsou P, Akogbeto M, Tanner M: Rapid Urban Malaria Appraisal (RUMA) IV: epidemiology of urban malaria in Cotonou (Benin). Malar J 2006, 5:45.

52. Yacoub S, Mohammed MJ, Ramsan M, Albonico M: Clinical predictors of malaria and other febrile illnesses in children under five on Pemba Island, Tanzania. Trop Doct 2005, 35:78-81.

53. Ogungbamigbe TO, Ojurongbe OO, Ogunro PS, Olowe OA, Elemile PO: Prevalence and transmission pattern of Plasmodium falciparum infection in Osogbo metropolis, southwest, Nigeria. Afr J Med Med Sci 2007, 36:305-310.

54. Patrick Kachur S, Schulden J, Goodman CA, Kassala H, Elling BF, Khatib RA, Causer LM, Mkikima S, Abdulla S, Bloland PB: Prevalence of malaria parasitemia among clients seeking treatment for fever or malaria at drug stores in rural Tanzania 2004. Trop Med Int Health 2006, 11:441-451.

55. Reyburn H, Ruanda J, Mwerinde O, Drakeley C: The contribution of microscopy to targeting antimalarial treatment in a low transmission area of Tanzania. Malar J 2006, 5:4.

56. Mens PF, van Amerongen A, Sawa P, Kager PA, Schallig HDFH: Molecular diagnosis of malaria in the field: development of a novel 1-step nucleic acid lateral flow immunoassay for the detection of all 4 human Plasmodium spp. and its evaluation in Mbita, Kenya. Diagn Microbio/ Infect Dis 2008, 61:421-427.

doi:10.1186/1475-2875-9-240

Cite this article as: D'Acremont et al:: Reduction in the proportion of fevers associated with Plasmodium falciparum parasitaemia in Africa: a systematic review. Malaria Journal 2010 9:240.

\section{Submit your next manuscript to BioMed Central and take full advantage of:}

- Convenient online submission

- Thorough peer review

- No space constraints or color figure charges

- Immediate publication on acceptance

- Inclusion in PubMed, CAS, Scopus and Google Scholar

- Research which is freely available for redistribution

Submit your manuscript at www.biomedcentral.com/submit
C Biomed Central 